\title{
Growth Mechanism of a Hybrid Structure Consisting of a Graphite Layer on Top of Vertical Carbon Nanotubes
}

\author{
Nicolo' Chiodarelli, ${ }^{1,2}$ Cigang Xu, ${ }^{3}$ Olivier Richard, ${ }^{1}$ Hugo Bender, ${ }^{1}$ \\ Alexander Klekachev, ${ }^{1,4}$ Mike Cooke, ${ }^{3}$ Marc Heyns, ${ }^{1,5}$ Stefan De Gendt, ${ }^{1,6}$ \\ Guido Groeseneken, ${ }^{1,2}$ and Philippe M. Vereecken ${ }^{1,7}$ \\ ${ }^{1}$ imec, Kapeldreef 75, 3001 Leuven, Belgium \\ ${ }^{2}$ Department of Electrical Engineering, K.U. Leuven, Kasteelpark Arenberg 1, 3001 Leuven, Belgium \\ ${ }^{3}$ Oxford Instruments Plasma Technology, North End, Yatton, Bristol BS49 4AP, UK \\ ${ }^{4}$ Department of Physics and Astronomy, K.U. Leuven, Celestijnenlaan 200D, 3001 Leuven, Belgium \\ ${ }^{5}$ Metallurgy and Materials Engineering Department, K.U. Leuven, Kasteelpark Arenberg 44, 3001 Leuven, Belgium \\ ${ }^{6}$ Chemistry Department, K.U. Leuven, Celestijnenlaan 200f, Bus 2404, 3001 Leuven, Belgium \\ ${ }^{7}$ Centre for Surface Chemistry and Catalysis (COK), K.U. Leuven, Kasteelpark Arenberg 23, 3001 Leuven, Belgium
}

Correspondence should be addressed to Philippe M. Vereecken, vereeck@imec.be

Received 14 October 2011; Accepted 3 June 2012

Academic Editor: Jianyu Huang

Copyright (C) 2012 Nicolo' Chiodarelli et al. This is an open access article distributed under the Creative Commons Attribution License, which permits unrestricted use, distribution, and reproduction in any medium, provided the original work is properly cited.

Graphene and carbon nanotubes (CNTs) are both carbon-based materials with remarkable optical and electronic properties which, among others, may find applications as transparent electrodes or as interconnects in microchips, respectively. This work reports on the formation of a hybrid structure composed of a graphitic carbon layer on top of vertical CNT in a single deposition process. The mechanism of deposition is explained according to the thickness of catalyst used and the atypical growth conditions. Key factors dictating the hybrid growth are the film thickness and the time dynamic through which the catalyst film dewets and transforms into nanoparticles. The results support the similarities between chemical vapor deposition processes for graphene, graphite, and CNT.

\section{Introduction}

Graphene and carbon nanotubes (CNTs) are carbon allotropes with similar and exceptional optical and electronic properties [1] which can find applications as electronic or optoelectronic devices such as interconnects $[2,3]$, transparent electrodes [4], photodiodes [5], photodetectors [6], or solar cells [7]. The similarity in their properties derives from the identical atomic structure consisting of sp2hybridized carbon atoms arranged in a hexagonal lattice and from the fact that the band structure of CNT is derived from that of graphene simply by confining the electronic wave functions to a smaller portion of a graphene plane [8].

Moreover, their manufacturing process is similar since CNT [9-12] and graphene [13-15] can be deposited via catalytic chemical vapor deposition (CVD) by exploiting the catalyzed decomposition reaction between a carbon precursor (acetylene for example) and a catalyst of the iron group $(\mathrm{Ni}, \mathrm{Co}$, or $\mathrm{Fe})$. More specifically, the catalyst provides active sites for adsorption and decomposition of the precursor molecule and a template to organize the adsorbed and/or dissolved carbon into graphitized structures. The main difference between the two processes lies in the shape of the template; that is, a nanoparticle in the case of onedimensional CNT or carbon nanofiber and a film (infinite large particle) in the case of two-dimensional graphene.

In this work, the link between graphene and CNT is further investigated by studying and explaining the formation mechanism of a hybrid structure consisting of graphitic layers on top of CNT. This peculiar structure is receiving increasing interest in the scientific community $[3,16-18]$ since it basically shows that an horizontal top electrode of 


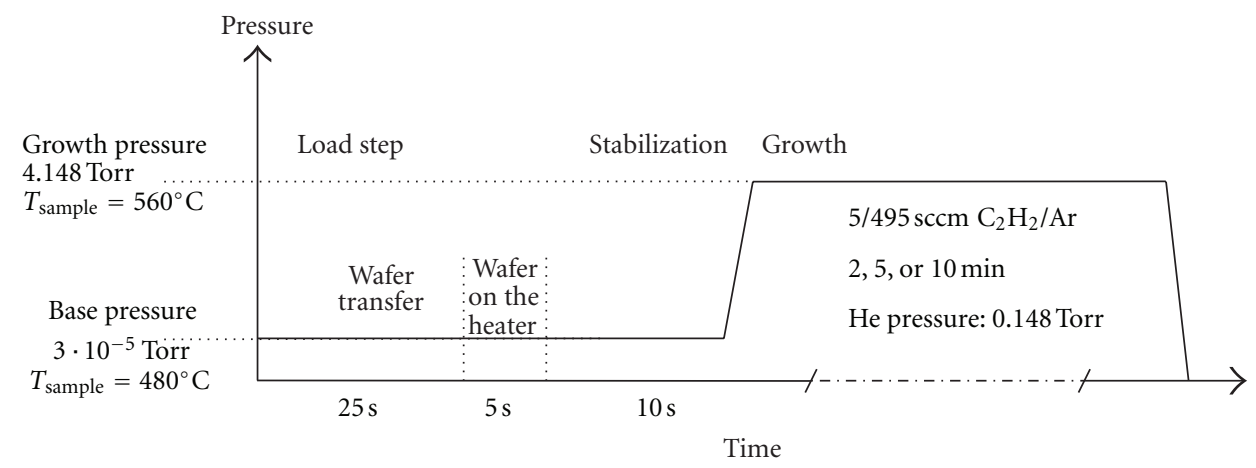

Figure 1: Scheme of growth recipes and tool operations. During the first load step of $30 \mathrm{~s}$, samples are transferred into the chamber at base pressure. A $10 \mathrm{~s}$ stabilization step is used for stabilizing sample temperature. The last growth step occurs in $\mathrm{C}_{2} \mathrm{H}_{2} / \mathrm{Ar}_{\mathrm{mixture}}(5 / 495 \mathrm{sccm})$ at a total pressure of 4.148 Torr and for 2, 5, or 10 minutes. Helium partial pressure is 0.148 Torr.

graphite can be deposited over vertical CNT in a single process step at low temperature whereas most of the times the two materials are deposited with independent stand-alone processes at high temperature. For instance, a horizontal top electrode in graphite could serve for interconnections [3] as a protective carbon cap for the CNT underneath or as selfformed large area electrode to contact the carbon nanotubes [18]. Clearly, the coexistence of both graphite and CNT in a unique process window would offer advantages in terms of manufacturability using a relatively inexpensive single-step process.

In this work, the key factors dictating the process window for growing the hybrid structure are identified in the film thickness, studied here in a relatively narrow range, and the time dynamic through which the catalyst film dewets and transforms into nanoparticles (also dependent, among other factors, on the film thickness). A based growth mechanism is identified for the growth of the hybrid structure on TiN as observed in [17] but not in [3]. Its formation appears catalyst independent since the hybrid structure has been observed for $\mathrm{Co}$ and $\mathrm{Ni}$ on TiN (this work and $[3,17]$ ), $\mathrm{Ni}$ on Ti [16], or for $\mathrm{Fe}$ on $\mathrm{SiO}_{2}$ [18]. Finally, based on the observations of catalyst debris near the base or inside the CNT, we agree with the qualitative idea that the CNTs underneath the structure form as a result of a catalyst in a viscous liquid-like state of high mobility over the underlying metallic substrate [17].

\section{Experimental}

Nickel and cobalt films of different thicknesses were deposited on a blanket $70 \mathrm{~nm}$ TiN/100 $\mathrm{nm} \mathrm{SiO} / 2 / p-S i$ stack by physical vapor deposition (PVD). The amount of material deposited by PVD was first calibrated on $\mathrm{SiO}_{2}$ test wafers and measured using Rutherford backscattering (RBS). The depth of the test films measured by RBS was $1.2410^{16}$ atoms $\cdot \mathrm{cm}^{-2}, 2 \cdot 10^{16}$ atoms $\cdot \mathrm{cm}^{-2}$ for nickel and $1.02 \cdot 10^{16}$ atoms $\cdot \mathrm{cm}^{-2}, 1.75 \cdot 10^{16}$ atoms $\cdot \mathrm{cm}^{-2}$ for cobalt, respectively. These data correspond to an equivalent thickness of about $1.3 \mathrm{~nm}$ and $2.2 \mathrm{~nm}$ for nickel and $1.1 \mathrm{~nm}$ and $1.9 \mathrm{~nm}$ for cobalt, respectively. Moreover, a film with an equivalent thickness of $5 \mathrm{~nm}$ was also included in the set of experiments.

In a typical growth experiment, a sample of each was loaded together. Pieces of 2 by $2 \mathrm{~cm}$ were placed next to each other in the center of a carrier wafer and processed in a plasma-lab CVD tool from Oxford Instrument consisting of a main deposition chamber with a heating stage of graphite connected to a load lock stage by a robot. Gasses are top injected into the main chamber via showerhead gas inlets in the top electrode.

As the exact sequence of tool operations during the growth experiments is important for the interpretation of the results, the growth recipe and the tool operations will be described in detail and are schematically represented in Figure 1 for clarity. All recipes begin with a load step where the samples are automatically transferred from the load lock into the main chamber in about $30 \mathrm{~s}$. As soon as the carrier wafer is in contact with the heater in the last $5 \mathrm{~s}$ of the load step, the sample temperature rapidly increases to approximately $T_{\mathrm{BP}}=480^{\circ} \mathrm{C}$, which is the sample temperature at the chamber base pressure of $3 \cdot 10^{-5}$ Torr.

For temperature stabilization, the samples were left for $10 \mathrm{~s}$ (stabilization step) in the chamber at base pressure. Then, the growth step begins and a $5 / 495 \mathrm{sccm} \mathrm{C}_{2} \mathrm{H}_{2} / \mathrm{Ar}$ mixture was introduced into the chamber up to the process pressure of 4.148 Torr. Helium was also present in the chamber at this stage at a partial pressure of 0.148 Torr. As a result of the pressure increase, the sample temperature rapidly increases to about $T_{\text {growth }}=560^{\circ} \mathrm{C}$. Note that, except for the loading and stabilization steps, no extra heat treatment or catalyst activation step was done.

For all experiments, the growth step consisted only of the operations described above, except for the fact that the growth time was varied for 2, 5, and 10 minutes. After growth, the samples were characterized by SEM and TEM. For TEM analysis samples were sonicated in isopropanol for 10 minutes and few droplets of the solution were deposited on a carbon lacey copper grid and dried. The analysis was done with a HRTEM Tecnai F30 operating at $200 \mathrm{kV}$ to limit damage to the CNT during beam exposure.

\section{Results}

Figure 2 shows the growth results for cobalt and nickel films with increasing film thickness after 5 minutes of growth time. Dense CNT mats were obtained for layers below $1.3 \mathrm{~nm}$, 


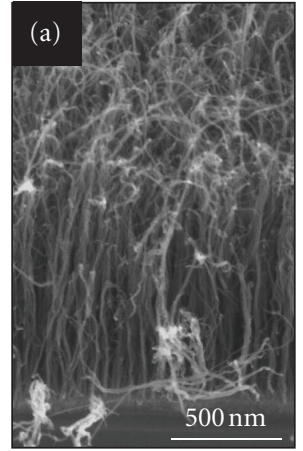

$1.1 \mathrm{~nm}$ Co

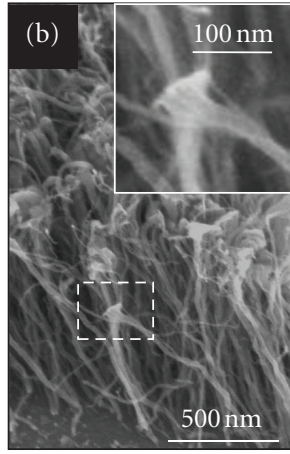

$1.3 \mathrm{~nm} \mathrm{Ni}$

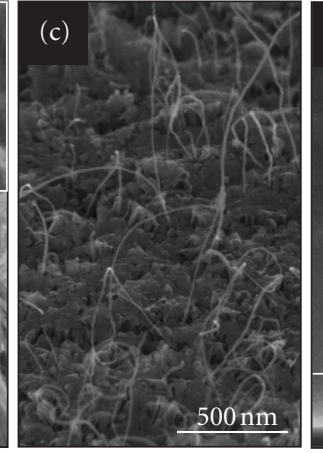

$1.9 \mathrm{~nm} \mathrm{Co}$

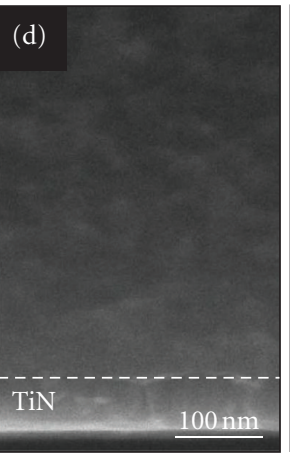

$2.2 \mathrm{~nm} \mathrm{Ni}$

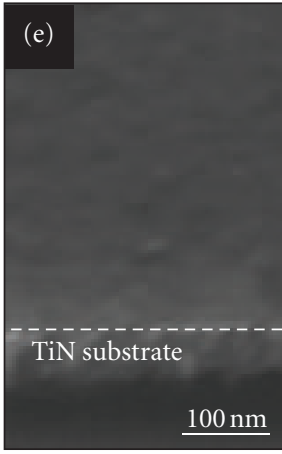

$5 \mathrm{~nm} \mathrm{Ni}$

CNT and graphite

top-layer

FIGURE 2: SEM pictures of different carbon nanostructures grown from thin nickel and cobalt films with different initial catalyst thickness in mixture of 5/495 sccm C2 $\mathrm{H}_{2} / \mathrm{Ar}$ for $5 \mathrm{~min}$ at 4.148 Torr. (a) CNT from $1.1 \mathrm{~nm} \mathrm{Co}$, (b) CNT from $1.3 \mathrm{~nm}$ Ni. The inset shows a CNT with a particularly tapered tip; (c) CNT with a top porous carbon layer grown from $1.9 \mathrm{~nm} \mathrm{Co}$; (d) and (e) no growth of carbon structures from $2.2 \mathrm{~nm}$ and $5 \mathrm{~nm} \mathrm{Ni}$.

while no CNTs were observed for films equal to and thicker than $2.2 \mathrm{~nm}$, under the presented growth conditions. For the intermediate film thickness of $1.9 \mathrm{~nm}$, CNT growth was also observed but an atypical layer can be distinguished on top of the CNT (Figure 2(c)). This top layer appears to be porous and some elongated CNTs extend through the pores. For a film thickness of $1.3 \mathrm{~nm}$, a peculiar tapered shape was found near the top of the CNT mat, showing a broadening towards a platform shape on top of the CNT (inset in Figure 2(b)).

To study the type of structures in more detail, TEM analysis was done on the 1.1-Co, 1.3-Ni, and 1.9-Co samples. In all cases (Figures 3 and 4), the structures consist of multiwalls CNT with diameters $10.8 \pm 3.4 \mathrm{~nm}$ for $1.1-\mathrm{Co}$, $13.1 \pm 5.1 \mathrm{~nm}$ for $1.3-\mathrm{Ni}$, and $12.7 \pm 5.0 \mathrm{~nm}$ for the $1.9-\mathrm{Co}$ samples as measured by SEM.

For the thinnest $1.1 \mathrm{~nm}$ film, cobalt nanoparticles are located mostly at the extremities of the tubes (Figure 3(a)) whereas only a few were found in the interior of the tube. Similarly, the 1.3-Ni sample had particles at the tube extremities but the amount of particles found inside the tube interior was larger in this case. Figure 3(d) shows a detail of the tapered platform-like shape which was observed with SEM at the tip of some CNT (inset of Figure 2(b)). As for the 1.9-Co sample, a crust has formed on top of the CNT but isolated to a single or a few interconnected CNT in contrast to the closed (porous) layer formed on the 1.9-Co sample in Figure 2(c).

In the 1.9-Co sample, the crusty top layer indeed connects the tips of all neighboring CNTs as confirmed by the TEM image in Figure 4(a). Underneath the crust, straight multiwall CNTs are present as shown at high magnification in Figures 4(b) and 4(c). Note that under different growth conditions, carbon nanofibers were found underneath similarly crusted top layers [19]. The fact that the CNTs are still connected to the top layer allows us to unambiguously locate the root of the tubes. From TEM, it follows that a cobalt nanoparticle sits at the base or root of the CNT. Arrows in Figure 4(a) indicate the particles in the tubes and a high-resolution image of a cobalt nanoparticle at the root of one CNT are shown in Figure 4(e).

For the 1.9-Co sample, nanoparticles are also in the core of some tubes (Figure 4(d)). In particular, comparing the inner particles among the samples, their number and size seem to increase according to the initial catalyst thickness: few and smaller nanoparticles for the 1.1-Co sample meanwhile numerous and larger nanoparticles in the $1.3-\mathrm{Ni}$ and 1.9-Co samples. Note that the interior "particles" can be quite elongated to rod-like structures (see Figures 3(e) and $4(d))$ and in some cases even long nanowires suggesting the formation of metal filaments during growth.

Figure 5 shows high-resolution TEM images of the toplayer for 1.9-Co samples. Multiple layers of graphene form the graphitic top layer or crust (Figures 5(a) and 5(b)). Even though the TEM shows only clearly the graphene sheets at the very surface, they appear to extend much further but their visualization is difficult because of overlapping. The global structure looks entangled without long range order (polycrystalline). Small and elongated nanoparticles are found right below the graphitic top-layer; that is, in the tapered transition region between the planar graphite layer and the vertical CNT. The tapered shape is then formed by graphitic encapsulation of these catalyst particles, some of which are trapped into this transition layer, others already in the CNT below.

Figure 6 shows the Raman spectra of the samples analyzed so far (from Figure 2 to Figure 5). The spectra are reproducible and representative since they appear identical for different spots over the same sample.

The samples with CNT only (1.1-Co) and with CNT and a graphitic layer on top (1.9-Co) exhibit identical Raman spectra. This is probably due not only to the fact that the spectra of graphite and MWCNT of large diameter have strong similarities but also depends on the penetration depth of the laser in our setup using a wavelength of $632 \mathrm{~nm}$ and a 

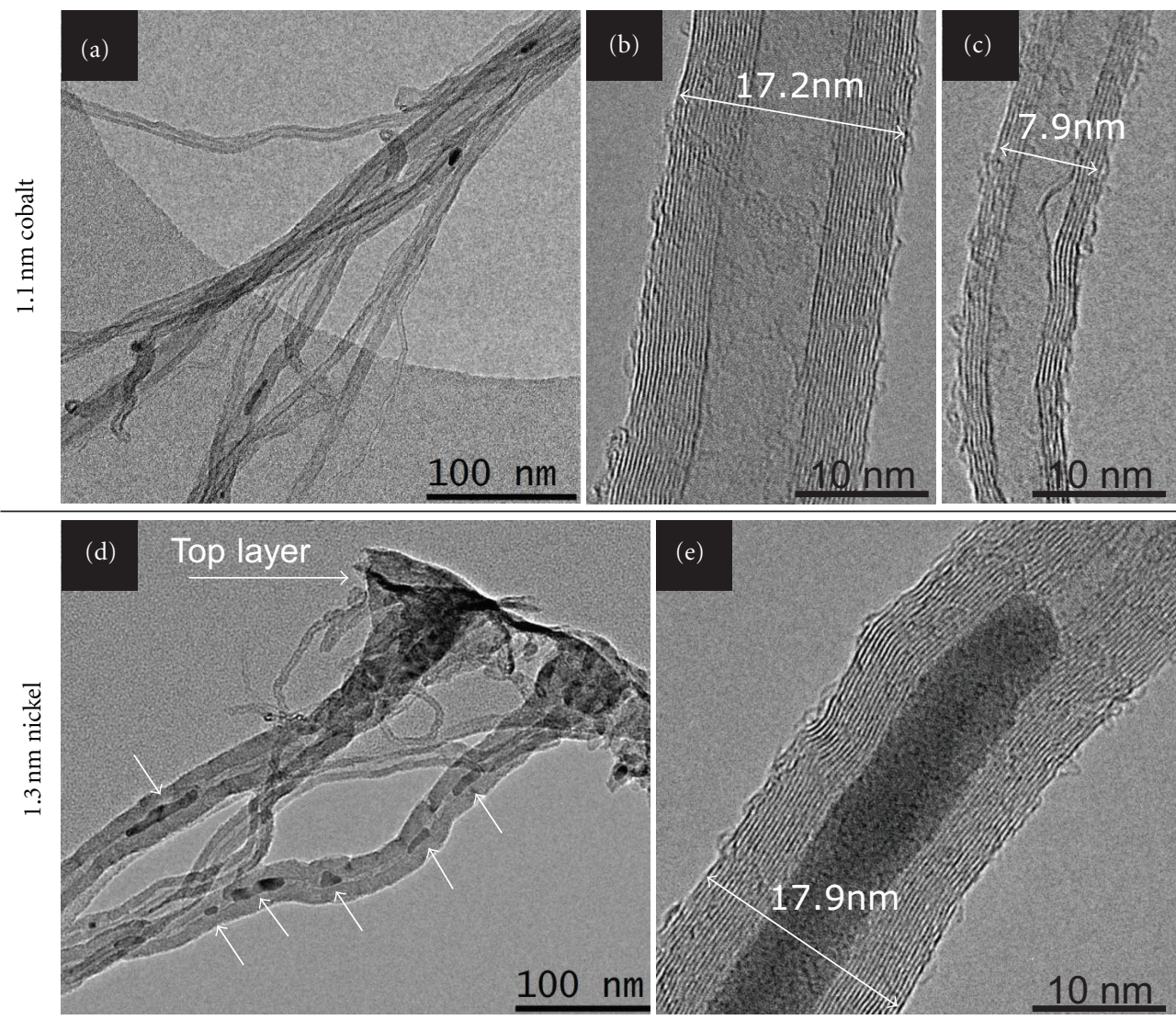

FIGURE 3: TEM of the carbon structures from $1.1 \mathrm{~nm}$ cobalt (top row, images (a), (b), (c)) and 1.3 nm nickel (bottom row, images (d) and (e)); (a) CNT at low magnification; (b) large CNT with 16 walls (b) small CNT with 6 walls; (d) carbon top layer at the CNT's tip; numerous particles inside the CNT highlighted by arrows; (e) CNT with a particularly large particle inside.

$1 \mu \mathrm{m}$ laser spot. For instance, a peak for silicon at $500 \mathrm{~cm}^{-1}$ is present in the spectra of the samples without CNT $(2.2 \mathrm{~nm}$ and $5 \mathrm{~nm}$ ) suggesting that the probed thickness is likely in the order of a hundred of nanometers as $\mathrm{SiO}_{2}$ is separated from the samples surface by a $70 \mathrm{~nm}$ thick layer of TiN. The top graphite layer, instead, is expected only few nanometers thick (from TEM) so large part of the Raman signal originates from the MWCNT underneath.

In all the samples, the typical features of graphitic materials are present in the first-order Raman spectra: that is, $D$ and $G$ bands at $\approx 1350 \mathrm{~cm}^{-1}$ and $\approx 1580 \mathrm{~cm}^{-1}$, respectively. Considering the pronounced shoulder in the $\mathrm{D}$ peak at about $1100 \mathrm{~cm}^{-1}$ and the fact that the $\mathrm{D}$ and $\mathrm{G}$ bands merge in the region between $1350 \mathrm{~cm}^{-1}$, and $\approx 1580 \mathrm{~cm}^{-1}$ it is concluded that the samples contain some degree of disorder. In fact, the TEM images of the graphite top layer (Figure 5) revealed a polycrystalline structure made of small domains of undulated fringes continuous over a length of few nanometers only. The edges of each domain can be considered as defects which contribute to the broadening of the $\mathrm{D}$ and $\mathrm{G}$ peaks as discussed in [20]. For graphitic materials with defects, a D' peak is also expected at $1620 \mathrm{~cm}^{-1}$ but it is not distinguishable here probably because buried in the G band as also expected for nanocrystalline graphite when small grains are present [21].
Similar arguments can also be applied to the samples with MWCNT even if broad G and D peaks are more typical because of the parallel contribution of several walls with different diameters. In particular, some degree of disorder in the structure of the tubes can be inferred from the shoulder at $2950 \mathrm{~cm}^{-1}$ [22]. Moreover, we noticed that the Raman spectra here reported are similar to those in [23] which were obtained for defective MWCNT and also to the spectra measured in previous works [24] on MWCNT grown at $470^{\circ} \mathrm{C}$ and $400^{\circ} \mathrm{C}$. In this latter case, the quality of the MWCNT was quantified by extracting the electrical resistivity. The result showed indeed that those MWCNT behaved as diffusive conductors for a length of few hundred of nanometers, namely, as a material with some degree of disorder or imperfections. The 2D peak [25] at $\approx 2700 \mathrm{~cm}^{-1}$ is an overtone of the $\mathrm{D}$ peak also present in graphite and CNT; however, for what mentioned above, it is believed not to originate from the graphite top layer.

The point of major interest for our discussion is that the $D$ and $G$ peaks also appear for samples without CNT (2.2-Ni and 5-Ni) and which looked inactive in the SEM of Figure 2(d) and Figure 2(e). In particular, since the G peak originates from sp2 carbon domains [23], we conclude that for these samples some graphitic carbon is deposited on the 


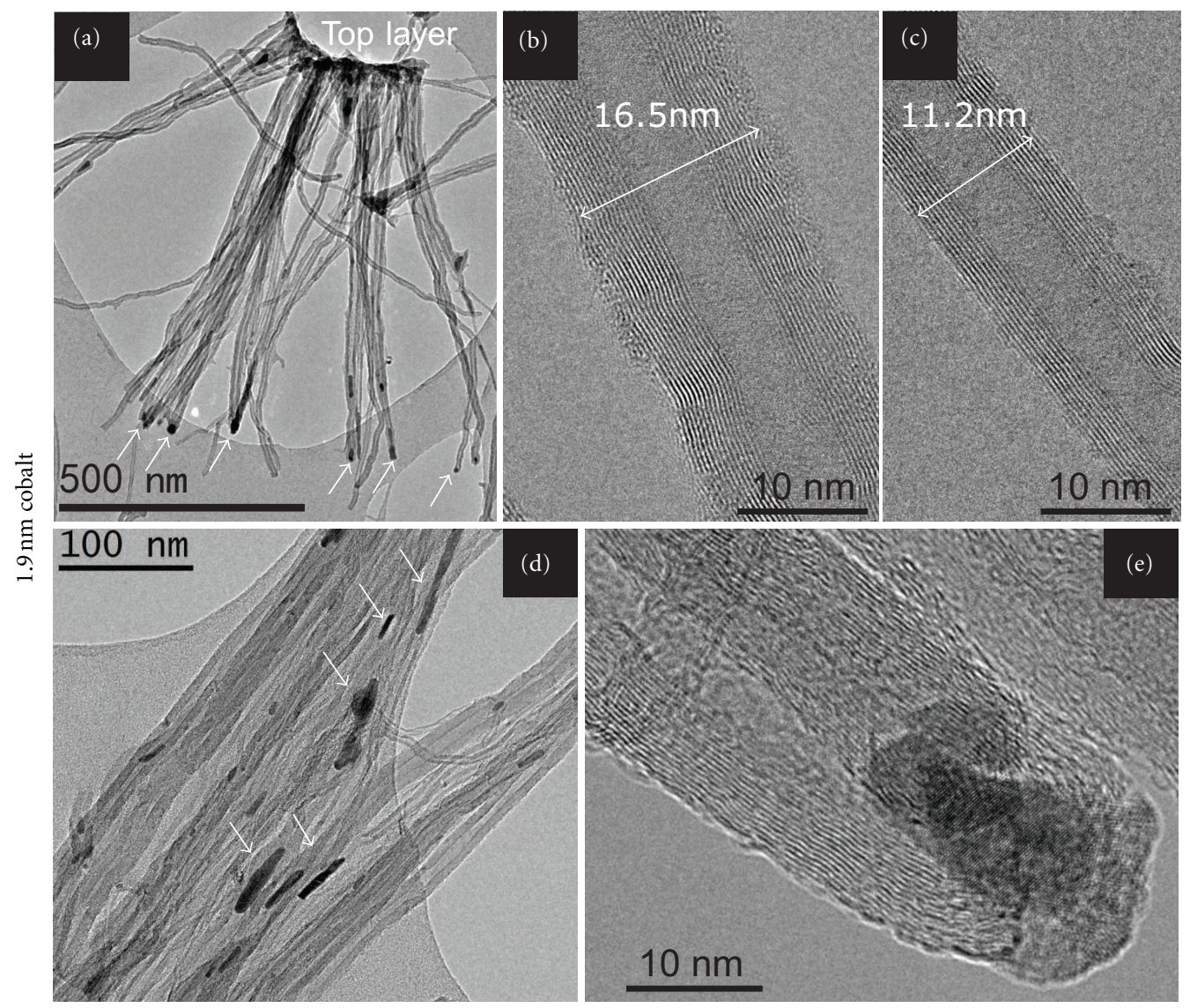

FIGURE 4: TEM of the carbon structures from $1.9 \mathrm{~nm}$ cobalt; (a) low-magnification image showing the presence of a large top layer on top of CNT; arrows indicate nanoparticles at the root of the CNT; (b) large CNT with 17 walls; (c) small CNT with 8 walls; (d) nanoparticles inside the CNTs as indicated by arrows; (e) nanoparticle at the tube root.
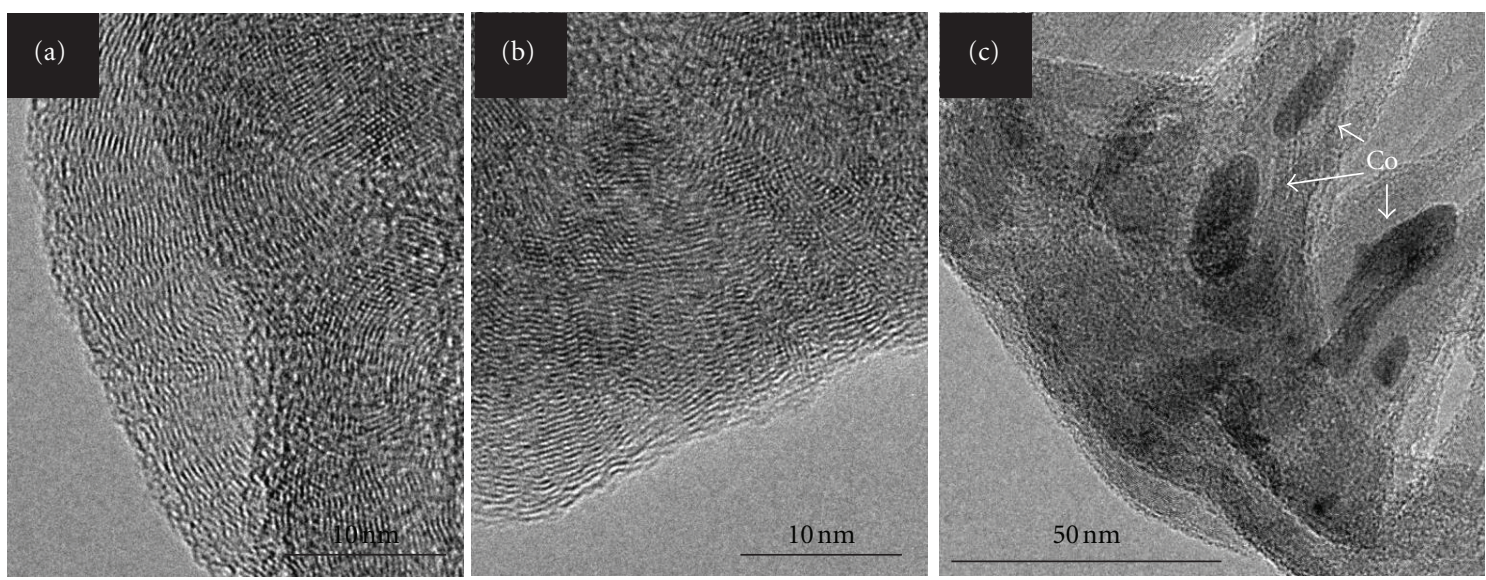

FIgURE 5: High-magnification TEM images of the top layer on the CNT grown from $1.9 \mathrm{~nm} \mathrm{Co.} \mathrm{(a)} \mathrm{and} \mathrm{(b):} \mathrm{graphitic} \mathrm{carbon} \mathrm{on} \mathrm{the} \mathrm{crest} \mathrm{of}$ the top layer; (c) lower-magnification picture showing elongated cobalt nanoparticles present right below the top layer.

catalyst in the early stage of the growth recipe even if CNT did not form.

The CNT length and consequently the height of the graphite top-layer increase linearly with growth time as shown in Figure $7(a)$. From the slope, a growth rate of $136 \mathrm{~nm} / \mathrm{min}$ was found. The intersection with the time axis suggests an incubation time of about $20 \mathrm{~s}$ before the graphite layer is lifted off by the CNT. The top-layer morphology did not change significantly with growth time as ascertained from the SEM images in Figures 7(b) and 7(c).

To assess the effect of pretreatment time [17] on thicker films, the $10 \mathrm{~s}$ stabilization step was extended to 2 minutes 


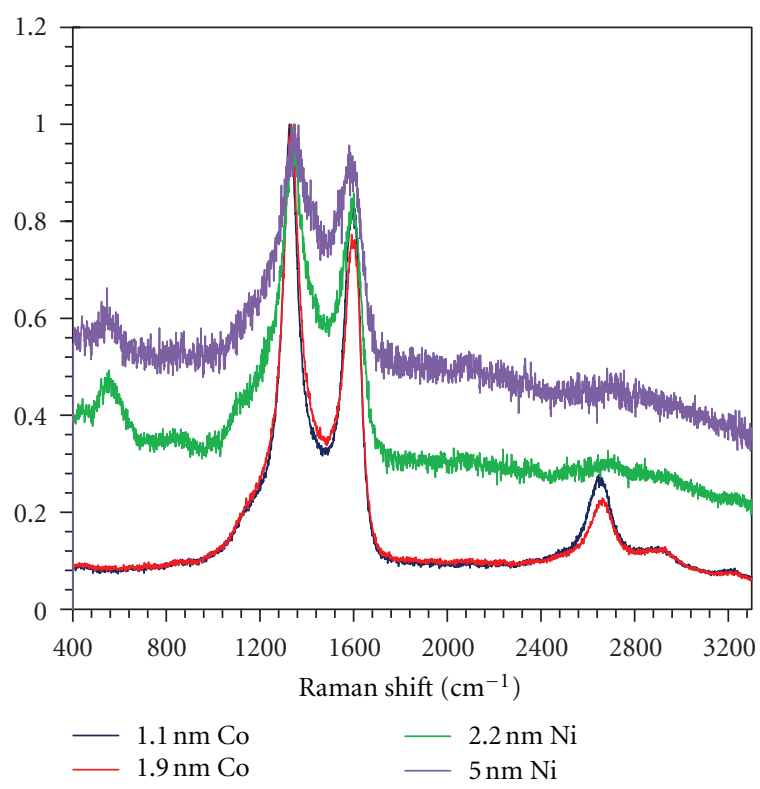

FIGURE 6: Raman spectra (normalized to the D band) of samples with nickel and cobalt films of different catalyst thickness grown in mixture of 5/495 sccm $\mathrm{C}_{2} \mathrm{H}_{2} / \mathrm{Ar}$ for $5 \mathrm{~min}$ at 4.148 Torr (same series as in Figure 2).

which is equivalent to a thermal pretreatment of $2 \mathrm{~min}$ and $10 \mathrm{~s}$ at $480^{\circ} \mathrm{C}$ in vacuum $\left(\approx 10^{-5}\right.$ Torr $)$ before the introduction of acetylene gas for growth. The remainder of the growth recipe was kept the same. The results for the 1.9Co and 2.2-Ni are presented in Figures 8(a) and 8(b). CNT growth was now observed in both cases without formation of any graphitic layer on top. The 5-Ni film still did not show any growth but after the thermal pretreatment was further extended to 10 minutes, CNTs under large patches of a graphitic top layer were achieved also on this sample (not shown here). Figure 8(c), instead, shows that CNT with a graphite top layer can also be grown in patterned structures using $1.3 \mathrm{~nm}$ of $\mathrm{Ni}$ and the same recipe without pretreatment used to grow the structures in Figure 2(b).

\section{Discussion}

The atypical structure observed in the 1.9-Co sample of Figure 4 basically consists of two components: a 2D graphitic top layer on top and a mat of 1D CNT underneath. It is here explained by the interaction between acetylene and the surface of the catalyst template which dynamically changes shape during the process; that is, from a planar film to distributed nanoparticles with possible formation of nanofilaments [28] as the first graphitic layer lifts off from the surface. Note that the term "nanoparticles" has to be seen in a broader sense in this case, namely, as a nucleation center for a CNT (e.g., extrusion in the metal film) which does not have a welldefined distribution in size and density as in the case of CNT growth with a controlled pretreatment or film dewetting step. The necessary conditions are discussed in more detail in the following.
For the decomposition reaction to occur, the catalyst template must be in its "active" state. In catalytic CVD of CNT, a pretreatment step is commonly needed to activate the nickel, cobalt, or iron catalyst. It is believed that the pure metallic form is the most active state for the decomposition of acetylene (access to the transition metal d-orbitals) [2931]. Reactive reducing gasses like $\mathrm{H}_{2}$ or $\mathrm{NH}_{3}$ are typically used to facilitate reduction of the native oxide, especially for those catalysts whose oxides are more difficult to reduce, like iron [29-31]. However, in the case of nickel and cobalt, the oxide reduction or removal is much easier which explains their higher reactivity at lower temperature $[12,31,32]$. For example, in [31], peaks in the XPS graph corresponding to metallic nickel were found at relatively low temperatures in vacuum.

In the first series of experiments, no dedicated pretreatment step was deliberately done. As CNT growth was still observed for the 1.1-Co and 1.3-Ni samples (Figures 2(a) and $2(\mathrm{~b})$ ), it can be concluded that the conditions used were sufficient for the activation of the catalyst. Activation may occur either in vacuum, namely, during the sequence of load, stabilization, and purge steps ( $40 \mathrm{~s}$ at $10^{-5}$ Torr of base pressure and $T_{\text {sample }}=480^{\circ} \mathrm{C}$ ) or, more likely, during the very early seconds of the growth step where acetylene or the $\mathrm{H}_{2}$ present as a product of its thermal decomposition can both help the reduction of $\mathrm{NiO}$.

Whether or not CNTs were formed (without a graphitic layer) was dependent on the pretreatment time. Indeed, it is generally accepted that catalyst nanoparticles are needed for CNT growth. Catalyst nanoparticles are typically formed by thermal [33-35] or plasma anneal $[10,36,37]$ of thin catalyst films. Because of the larger energy available at high temperatures, the catalyst atoms become mobile which can lead dewetting of the film [38].

The results found here indicate that the time scale at which a film dewets and transforms into nanoparticles is strongly related to the initial film thickness. In fact, only the thinnest films $(<1.3 \mathrm{~nm})$ gave CNT when a short (process intrinsic) pretreatment of $40 \mathrm{~s}$ was done (Figures 2, 3, and 4). In the case of $1.1 \mathrm{~nm} \mathrm{Co}$, this is because either the film was thin enough to break with a 40 s pretreatment either because it was already discontinuous after deposition. The $1.3 \mathrm{~nm}$ Ni film, instead, showed small graphitic patches after the 40 s pretreatment. Since a graphitized top layer was observed for the same film in patterned structures (see Figure $7(\mathrm{c})$ ), this film must have been initially continuous (see $[26,27]$ for details about the patterned structures). In this case, film breakup occurs during the $40 \mathrm{~s}$ pretreatment step, although partially complete.

Thicker films either gave CNT with a graphite layer on top $(1.9-\mathrm{Co})$ or were inactive $(2.2-\mathrm{Ni}$ and $5-\mathrm{Ni})$ with only a 40 s pretreatment. However, when the vacuum anneal pretreatment time was increased to $2 \mathrm{~min}$ also the 1.9-Co and 2.2-Ni yielded CNT without the graphitic layer indicating that the catalyst film was completely transformed into nanoparticles (Figures 8(a) and 8(b)). Even the $5 \mathrm{~nm}$ thick Ni film could eventually be broken into large patches after $10 \mathrm{~min}$ of vacuum anneal. Note that the assistance of plasma and reducing gasses may aid the dewetting of thick films [9]. 
(a)

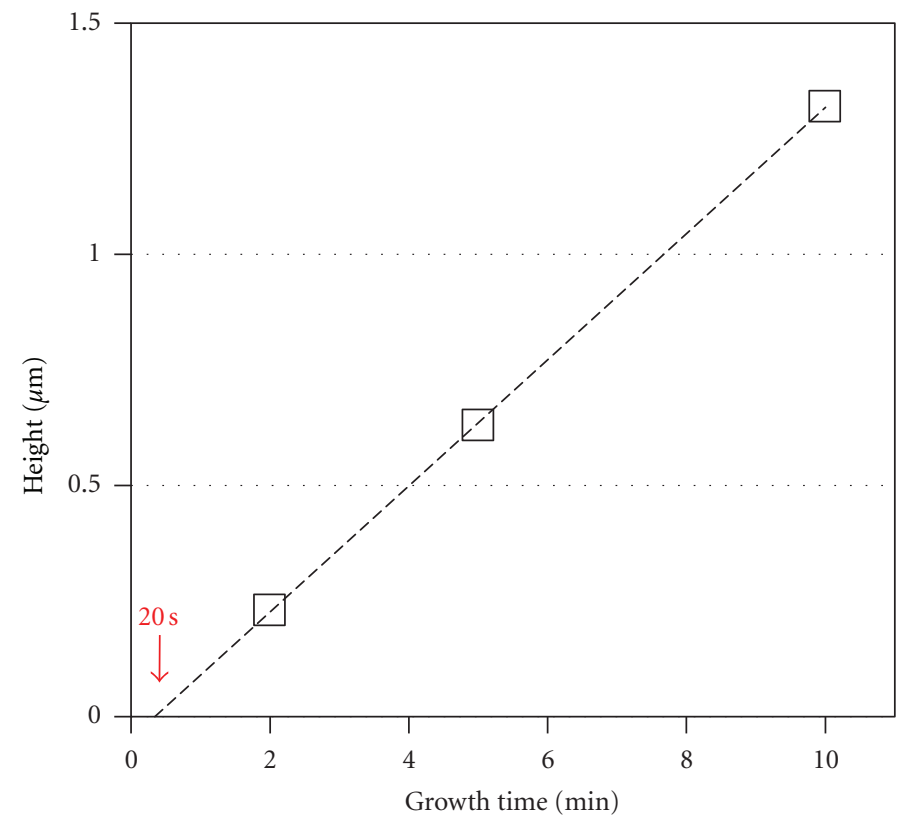

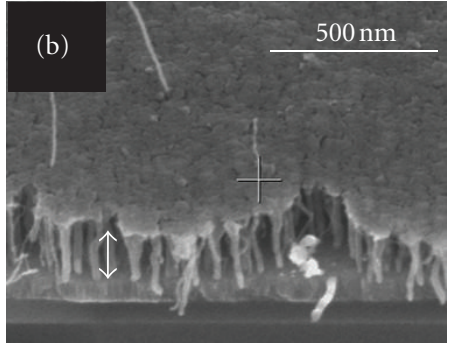

Growth time: $2^{\prime}$

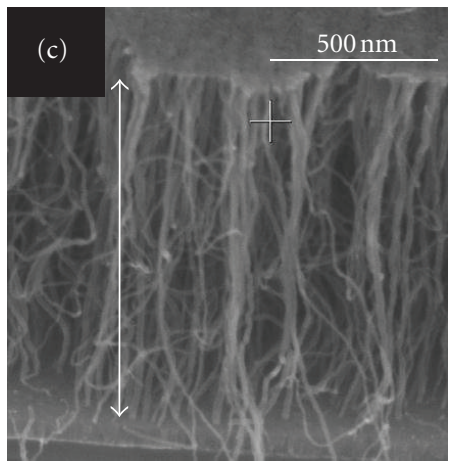

Growth time: $10^{\prime}$

Figure 7: (a) Height of the CNT graphite hybrid structure from $1.9 \mathrm{~nm}$ Co in function of the growth time. The extrapolated line intersects the $x$-axis at $20 \mathrm{~s}$ (incubation time); (b) and (c) SEM pictures of CNT with graphite top layer grown for, respectively, 2 and 10 minutes. Acetylene was introduced in the chamber at time $t=0$ of the graph.
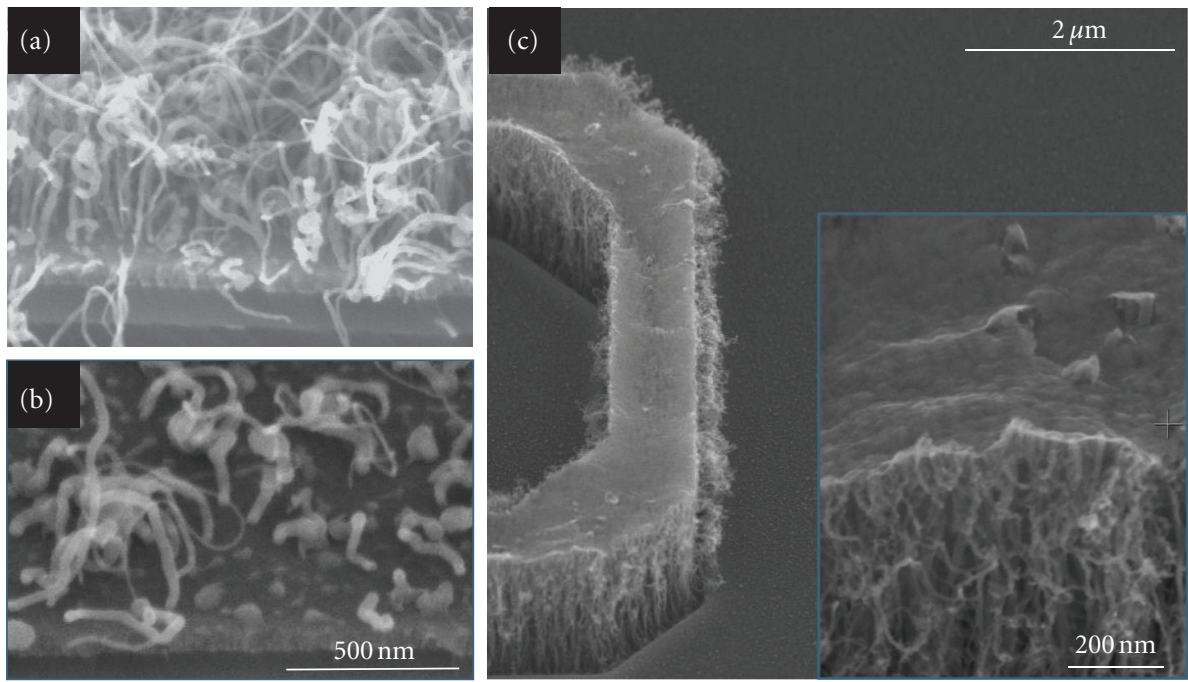

Figure 8: (a) CNT grown from a $1.9 \mathrm{~nm}$ Co film and (b) from a $2.2 \mathrm{~nm}$ Ni film after 2 minute thermal pretreatment (same scale). For both, thermal pretreatment of $2^{\prime} 10 \mathrm{~s}$ in vacuum $\left(T_{\text {sample }}=480^{\circ} \mathrm{C}\right)$ and growth step in $5 / 10 / 495 \mathrm{sccm} \mathrm{C}_{2} \mathrm{H}_{2} / \mathrm{He} / \mathrm{Ar}$ at $4.148 \mathrm{Torr}$ for 5 min $\left(T_{\text {sample }}=560^{\circ} \mathrm{C}\right)$; (c) top layer visible on top of patterned structures with $1.3 \mathrm{~nm} \mathrm{Ni}$ deposited. No pretreatment was used in this case. Details on the patterned structures of $c$ are in $[26,27]$.

Thus far, we have confirmed the notion that CNTs are formed only when nanoparticles are present before the growth step commences. Now, we will discuss the intermediate phase where the carbon precursor gas (acetylene) is introduced when the catalyst film is still, at least partly, intact. Indeed, the reshaping dynamic for films thicker than $1.3 \mathrm{~nm}$ occurs on a time scale of minutes. Hence, when acetylene is introduced in the chamber immediately after the load and stabilization steps, as for Figure 2, little time is left for these films to transform into nanoparticles. In the $2.2 \mathrm{~nm}$ and $5 \mathrm{~nm} \mathrm{Ni}$ films, no growth at all was observed after $40 \mathrm{~s}$ of pretreatment. As the catalyst is active, though, these layers 
have been covered by a graphitic coating as confirmed by the presence of $D$ and $G$ peaks in the Raman spectra (Figure 6).

The $1.9 \mathrm{~nm}$ Co sample has a thickness intermediate to the extreme cases mentioned above. It is sufficiently thick to be continuous after its deposition but still sufficiently thin to fragment into nanoparticles when the pretreatment time allows for it. The incubation time of $20 \mathrm{~s}$ observed in Figure 7(a) indicates that CNT growth does not occur immediately after the introduction of acetylene. Hence, the film retains its $2 \mathrm{D}$ film-like shape during the whole load and stabilization steps and at least for the first seconds of the growth steps. Because the catalyst is active, the decomposition of acetylene can occur and a graphitic layer is formed on the surface of the film at this stage, similarly to what happens in catalyzed deposition processes of graphene [39].

At this stage, film fragmentation proceeds and after about $20 \mathrm{~s}$ CNT growth can start. Consequently, the top layer is lifted by the CNT structures growing underneath. Theoretical calculations showed that graphitized structures have low adhesion to nickel (100) [40] and $\mathrm{Ni}$ (111) [41] surfaces which supports the idea that the fragmentation of the underlying catalyst film can occur without being hampered by the graphite top layer. Note also that CNT growth might start either from nanoparticles as well as from sharp protrusions which form during the dynamic reshaping of the catalyst film under the graphite layer. In this latter case, metal filaments encapsulated in graphene sheets are observed at the bottom of the CNT like in Figure 4(a).

This seems a direct consequence of the fact that catalyst saturated with carbon appears is in a viscous semiliquid state $[42,43]$ suggesting facilitated reshaping. Metal atoms in this phase are much more mobile than in pure metal which leads the catalyst to easily deform into elongated shapes [28]. For example, elongation and reshaping of catalyst nanoparticles has been observed at the initial stages of CNT growth with in situ TEM $[44,45]$. Hence, reshaping of the catalyst template can be facilitated by carbon; that is, after introducing acetylene in the chamber. Furthermore, the semi liquid carbon-saturated catalyst state explains how the CNTs end up partly filled with metal especially near the top layer where growth started.

A few remarks should be pointed out regarding the catalyst particles formed during this dynamic process. It is often mentioned that the CNT diameter closely matches that of the original nanoparticle. In particular, for MWCNT, a $1: 1$ correlation between particle's and CNT's diameters was observed in [46, 47] but not [48]. This rule does not apply here as the amount of catalyst found inside the CNT as debris is large and cannot originate from a single nanoparticle with the same diameter of the tube. It seems more plausible that CNTs start growing from particular nucleation points on a larger patch of catalyst. Because of its semiliquid state, the excess of metal is encapsulated in the final CNT and appears in its interior as nanoparticles. This is a major difference compared to most of reported literature data on CNT where the growth recipes had dedicated pretreatments to properly break the catalyst into well-defined nanoparticles.
As catalyst is observed at the CNT root as well as right below the top layer (Figure 5(c)), the CNT growth mechanism is not evident. Nevertheless, the fact that several elongated particles are observed at the CNT root suggests a strong catalyst substrate interaction sufficient to maintain the catalyst, or part of it, anchored to the substrate. The remaining excess of catalyst is dispersed along the entire tube's length as debris. This picture would lead us to propose a base growth mechanism as observed in literature for the Fe-TiN system [10] or for Ni-TiN systems [49-51]. The presence of pores in the top layer could suggest that these are the preferred channels through which the gas can reach the bottom substrate for feeding the catalyst reaction at the CNT base.

Our results have striking similarities with [3, 16-18]. Although the authors investigated the process window for different catalyst thickness and growth temperatures, a similar trend to that reported in Figure 2 can be deduced. In particular, a transition between CNT growth only, the growth of a graphite top layer on CNT, and no growth at all is observed when increasing catalyst thickness $[16,18]$. The process window for the hybrid graphite/CNT structure strongly depends on the capability of the catalyst to retain its film shape when the gas precursor is inserted into the chamber and to dewet during the subsequent instants. Hence, at high temperature, thicker catalyst films or growth recipes without pretreatments [3, 16-18] should be used to guarantee the formation of the graphite layer before the film dewets. At lower temperature [17], instead, thinner films or mild pretreatments can be used so to increase the catalyst mobility and guarantee that after some time the film dewets yielding CNT.

\section{Conclusions}

The growth of an atypical structure composed of CNT with a graphite top layer has been observed and studied. To summarize, the following four conditions have to be satisfied for the formation of this type of structure.

(1) The catalyst has to be active.

(2) The catalyst must initially be a continuous film.

(3) The carbon source has to be introduced before the catalyst has reshaped into nanoparticles.

(4) The thickness of the catalyst layer and the growth conditions have to be tuned such that the catalyst film retains its $2 \mathrm{D}$ shape during the first seconds of the growth but then it is sufficiently mobile to dynamically evolve into distributed nanoparticles.

The first three conditions allow for the formation of a graphitic layer on top of the catalyst film in a similar way to what reported in literature for catalytic deposition processes of graphite/graphene. When also the fourth condition is satisfied, then nanoparticles or patches of catalyst can reshape underneath the graphitic top layer to initiate CNT growth. Consequently, the top layer is lifted up by the underlying CNT [17]. In this phase, excess of catalyst can break and remain in the tubes interior as particles. 
Satisfying conditions 2, 3, and 4 all together is not straightforward as the dynamic a film transforms into nanoparticles was found to largely depend on the initial film thickness and temperature used. Very thin films, in fact, either are discontinuous after deposition so that condition 2 cannot be satisfied or break very quickly making condition 3 difficult to satisfy experimentally. Very thick films, instead, may not break at all making condition 4 incomplete. This explains the narrow process window for film thickness in which CNT and the graphite top layer are observed (Figure 2) under the growth conditions used: $1.9 \mathrm{~nm}$ for cobalt blanket films and $1.3 \mathrm{~nm}$ for $\mathrm{Ni}$ patterned samples. Note that this type of structure was observed for both nickel and cobalt but other catalysts might possibly be used probably in a different process window.

The result reported strength the clear correlation between deposition process of graphite and CNT. By improving the control on the dynamic of catalyst breakage and most likely by carefully tuning the growth conditions, it could be possible to achieve a well-defined transition between the growth of graphene or graphite on top of CNT. Such hybrid structures might be of relevant importance for electronic and optical applications such as carbon-based interconnects, field emitters, sensors, NEMs, and optical devices.

\section{Acknowledgments}

The authors would like to thank Francesca Clemente for support with Raman spectroscopy. The EU projects CARBonCHIP (NMP4-CT-2006-016475), TECHNOTUBES (NMP2-LA-2009-228579), and the METACEL project (IWTSBO project 060031) funded by IWT-Vlaanderen are acknowledged for financial support. Nicolo' Chiodarelli has this current address (CEA LITEN/DTNM/LCRE, 17 rue des Martyrs, 38054 Grenoble, France).

\section{References}

[1] P. Avouris, Z. Chen, and V. Perebeinos, "Carbon-based electronics," Nature Nanotechnology, vol. 2, no. 10, pp. 605615, 2007.

[2] N. Chiodarelli, S. Masahito, Y. Kashiwagi et al., "Measuring the electrical resistivity and contact resistance of vertical carbon nanotube bundles for application as interconnects," Nanotechnology, vol. 22, no. 8, Article ID 085302, 2011.

[3] D. Kondo, S. Sato, and Y. Awano, "Self-organization of novel carbon composite structure: graphene multi-layers combined perpendicularly with aligned carbon nanotubes," Applied Physics Express, vol. 1, no. 7, Article ID 074003, 2008.

[4] S. Bae, H. Kim, Y. Lee et al., "Roll-to-roll production of 30-inch graphene films for transparent electrodes," Nature Nanotechnology, vol. 5, no. 8, pp. 574-578, 2010.

[5] J. Lefebvre, D. G. Austing, J. Bond, and P. Finnie, "Photoluminescence imaging of suspended single-walled carbon nanotubes," Nano Letters, vol. 6, no. 8, pp. 1603-1608, 2006.

[6] M. Freitag, Y. Martin, J. A. Misewich, R. Martel, and P. Avouris, "Photoconductivity of single carbon nanotubes," Nano Letters, vol. 3, no. 8, pp. 1067-1071, 2003.

[7] J. Wei, Y. Jia, Q. Shu et al., "Double-walled carbon nanotube solar cells," Nano Letters, vol. 7, no. 8, pp. 2317-2321, 2007.
[8] J. W. Mintmire and C. T. White, "Electronic and structural properties of carbon nanotubes," Carbon, vol. 33, no. 7, pp. 893-902, 1995.

[9] M. Chhowalla, K. B. K. Teo, C. Ducati et al., "Growth process conditions of vertically aligned carbon nanotubes using plasma enhanced chemical vapor deposition," Journal of Applied Physics, vol. 90, no. 10, pp. 5308-5317, 2001.

[10] S. Esconjauregui, B. C. Bayer, M. Fouquet et al., "Growth of high-density vertically aligned arrays of carbon nanotubes by plasma-assisted catalyst pretreatment," Applied Physics Letters, vol. 95, no. 17, Article ID 173115, 2009.

[11] G. D. Nessim, A. J. Hart, J. S. Kim et al., "Tuning of verticallyaligned carbon nanotube diameter and areal density through catalyst pre-treatment," Nano Letters, vol. 8, no. 11, pp. 35873593, 2008.

[12] M. Cantoro, S. Hofmann, C. Mattevi et al., "Plasma restructuring of catalysts for chemical vapor deposition of carbon nanotubes," Journal of Applied Physics, vol. 105, no. 6, Article ID 064304, 2009.

[13] S. Kumar, N. McEvoy, T. Lutz et al., "Low temperature graphene growth," ECS Transactions, vol. 19, no. 5, pp. 175$181,2009$.

[14] A. Reina, X. Jia, J. Ho et al., "Large area, few-layer graphene films on arbitrary substrates by chemical vapor deposition," Nano Letters, vol. 9, no. 1, pp. 30-35, 2009.

[15] K. S. Kim, Y. Zhao, H. Jang et al., "Large-scale pattern growth of graphene films for stretchable transparent electrodes," Nature, vol. 457, no. 7230, pp. 706-710, 2009.

[16] C. C. Chuang, W. L. Liu, W. J. Chen, and J. H. Huang, "Growth of graphite film over the tops of vertical carbon nanotubes using $\mathrm{Ni} / \mathrm{Ti} / \mathrm{Si}$ substrate," International Journal of Minerals, Metallurgy and Materials, vol. 16, no. 1, pp. 128-134, 2009.

[17] V. Jousseaume, J. Cuzzocrea, N. Bernier, and V. T. Renard, "Few graphene layers/carbon nanotube composites grown at complementary-metal-oxide-semiconductor compatible temperature," Applied Physics Letters, vol. 98, no. 12, Article ID 123103, 2011.

[18] S. Das, R. Seelaboyina, V. Verma et al., "Synthesis and characterization of self-organized multilayered graphene-carbon nanotube hybrid films," Journal of Materials Chemistry, vol. 21, no. 20, pp. 7289-7295, 2011.

[19] A. Romo-Negreira, D. J. Cott, A. S. Verhulst et al., "Growth and integration of high-density CNT for BEOL interconnects," in Proceedings of the Materials Research Society Symposium Proceedings, vol. 1079, 2008.

[20] A. C. Ferrari, "Raman spectroscopy of graphene and graphite: disorder, electron-phonon coupling, doping and nonadiabatic effects," Solid State Communications, vol. 143, no. 1-2, pp. 4757, 2007.

[21] A. C. Ferrari and J. Robertson, "Interpretation of Raman spectra of disordered and amorphous carbon," Physical Review $B$, vol. 61, no. 20, pp. 14095-14107, 2000.

[22] M. A. Pimenta, G. Dresselhaus, M. S. Dresselhaus, L. G. Cançado, A. Jorio, and R. Saito, "Studying disorder in graphite-based systems by Raman spectroscopy," Physical Chemistry Chemical Physics, vol. 9, no. 11, pp. 1276-1291, 2007.

[23] Y. A. Kim, T. Hayashi, K. Osawa, M. S. Dresselhaus, and M. Endo, "Annealing effect on disordered multi-wall carbon nanotubes," Chemical Physics Letters, vol. 380, no. 3-4, pp. 319-324, 2003.

[24] N. Chiodarelli, Integration of carbon nanotubes as future interconnects for sub-32 nm technologies [Ph.D. thesis], Katholieke Universiteit Leuven, Leuven, Belgium, 2011. 
[25] M. S. Dresselhaus, G. Dresselhaus, R. Saito, and A. Jorio, "Raman spectroscopy of carbon nanotubes," Physics Reports, vol. 409, no. 2, pp. 47-99, 2005.

[26] N. Chiodarelli, K. Kellens, D. J. Cott et al., "Integration of vertical carbon nanotube bundles for interconnects," ECS Transactions, vol. 19, no. 24, pp. 11-24, 2009.

[27] N. Chiodarelli, Y. Li, D. J. Cott et al., "Integration and electrical characterization of carbon nanotube via interconnects," Microelectronic Engineering, vol. 88, no. 5, pp. 837-843, 2011.

[28] X. Chen, R. Wang, J. Xu, and D. Yu, "TEM investigation on the growth mechanism of carbon nanotubes synthesized by hot-filament chemical vapor deposition," Micron, vol. 35, no. 6, pp. 455-460, 2004.

[29] C. Mattevi, C. T. Wirth, S. Hofmann et al., "In-situ Xray photoelectron spectroscopy study of catalyst-support interactions and growth of carbon nanotube forests," Journal of Physical Chemistry C, vol. 112, no. 32, pp. 12207-12213, 2008.

[30] A. Okita, A. Ozeki, Y. Suda et al., "Analysis of oxidation state of multilayered catalyst thin films for carbon nanotube growth using plasma-enhanced chemical vapor deposition," Japanese Journal of Applied Physics, Part 1, vol. 45, no. 10 B, pp. 8323$8329,2006$.

[31] S. Hofmann, R. Blume, C. T. Wirth et al., "State of transition metal catalysts during carbon nanotube growth," Journal of Physical Chemistry C, vol. 113, no. 5, pp. 1648-1656, 2009.

[32] A. M. Cassell, Q. Ye, B. A. Cruden et al., "Combinatorial chips for optimizing the growth and integration of carbon nanofibre based devices," Nanotechnology, vol. 15, no. 1, pp. 9-15, 2004.

[33] E. Terrado, I. Tacchini, A. M. Benito, W. K. Maser, and M. T. Martínez, "Optimizing catalyst nanoparticle distribution to produce densely-packed carbon nanotube growth," Carbon, vol. 47, no. 8, pp. 1989-2001, 2009.

[34] S. Hermann, R. Ecke, S. Schulz, and T. Gessner, "Controlling the formation of nanoparticles for definite growth of carbon nanotubes for interconnect applications," Microelectronic Engineering, vol. 85, no. 10, pp. 1979-1983, 2008.

[35] S. Pisana, M. Cantoro, A. Parvez, S. Hofmann, A. C. Ferrari, and J. Robertson, "The role of precursor gases on the surface restructuring of catalyst films during carbon nanotube growth," Physica E, vol. 37, no. 1-2, pp. 1-5, 2007.

[36] A. Malesevic, H. Chen, T. Hauffman, A. Vanhulsel, H. Terryn, and C. V. Haesendonck, "Study of the catalyst evolution during annealing preceding the growth of carbon nanotubes by microwave plasma-enhanced chemical vapour deposition," Nanotechnology, vol. 18, no. 45, Article ID 455602, 2007.

[37] S. C. Chang, T. C. Lin, T. S. Li, and S. H. Huang, "Carbon nanotubes grown from nickel catalyst pretreated with $\mathrm{H}_{2} / \mathrm{N}_{2}$ plasma," Microelectronics Journal, vol. 39, no. 12, pp. 1572$1575,2008$.

[38] Y. Wang, Z. Luo, B. Li et al., "Comparison study of catalyst nanoparticle formation and carbon nanotube growth: support effect," Journal of Applied Physics, vol. 101, no. 12, Article ID 124310, 2007.

[39] J. Lahiri, T. Miller, L. Adamska, I. I. Oleynik, and M. Batzill, "Graphene growth on $\mathrm{Ni}(111)$ by transformation of a surface carbide," Nano Letters, vol. 11, no. 2, pp. 518-522, 2011.

[40] H. Amara, C. Bichara, and F. Ducastelle, "Interaction of carbon clusters with $\mathrm{Ni}\left(\begin{array}{lll}1 & 0 & 0\end{array}\right)$ : application to the nucleation of carbon nanotubes," Surface Science, vol. 602, no. 1, pp. 7783, 2008.

[41] H. Amara, C. Bichara, and F. Ducastelle, "Formation of carbon nanostructures on nickel surfaces: a tight-binding grand canonical Monte Carlo study," Physical Review B, vol. 73, no. 11, Article ID 113404, pp. 1-4, 2006.

[42] A. R. Harutyunyan, T. Tokune, and E. Mora, "Liquid as a required catalyst phase for carbon single-walled nanotube growth," Applied Physics Letters, vol. 87, no. 5, Article ID 051919, 2005.

[43] A. R. Harutyunyan, E. Mora, T. Tokune et al., "Hidden features of the catalyst nanoparticles favorable for single-walled carbon nanotube growth," Applied Physics Letters, vol. 90, no. 16, Article ID 163120, 2007.

[44] S. Helveg, C. López-Cartes, J. Sehested et al., "Atomic-scale imaging of carbon nanofibre growth," Nature, vol. 427, no. 6973, pp. 426-429, 2004.

[45] S. Hofmann, R. Sharma, C. Ducati et al., "In situ observations of catalyst dynamics during surface-bound carbon nanotube nucleation," Nano Letters, vol. 7, no. 3, pp. 602-608, 2007.

[46] A. Romo-Negreira, D. J. Cott, S. De Gendt, K. Maex, M. M. Heyns, and P. M. Vereecken, "Electrochemical tailoring of catalyst nanoparticles for CNT spatial-dimension control," Journal of the Electrochemical Society, vol. 157, no. 3, pp. K47K51, 2010.

[47] A. R. Harutyunyan, T. Tokune, E. Mora, J. W. Yoo, and A. J. Epstein, "Evolution of catalyst particle size during carbon single walled nanotube growth and its effect on the tube characteristics," Journal of Applied Physics, vol. 100, no. 4, Article ID 044321, 2006.

[48] C. Lu and J. Liu, "Controlling the diameter of carbon nanotubes in chemical vapor deposition method by carbon feeding," Journal of Physical Chemistry B, vol. 110, no. 41, pp. 20254-20257, 2006.

[49] J. B. A. Kpetsu, P. Jedrzejowski, C. Côté et al., "Influence of Ni catalyst layer and TiN diffusion barrier on carbon nanotube growth rate," Nanoscale Research Letters, vol. 5, no. 3, pp. 539$544,2010$.

[50] W. P. Wang, H. C. Wen, S. R. Jian et al., "The effects of hydrogen plasma pretreatment on the formation of vertically aligned carbon nanotubes," Applied Surface Science, vol. 253, no. 23, pp. 9248-9253, 2007.

[51] S. R. Jian, "Effects of $\mathrm{H}_{2}$ plasma pretreated Ni catalysts on the growth of carbon nanotubes," Materials Chemistry and Physics, vol. 115, no. 2-3, pp. 740-743, 2009. 

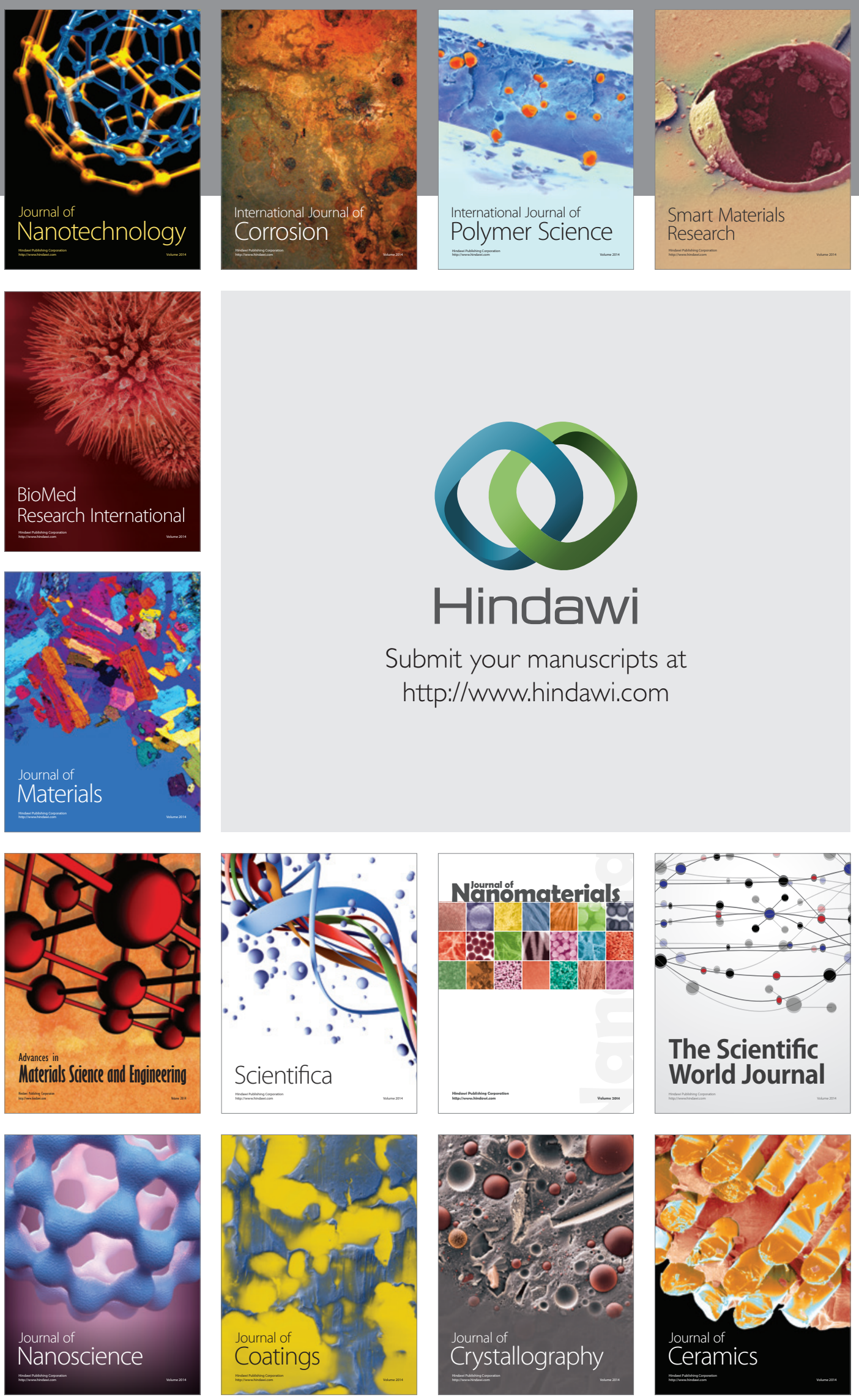

The Scientific World Journal

Submit your manuscripts at

http://www.hindawi.com

\section{World Journal}

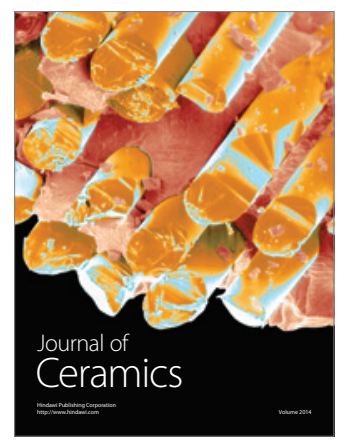

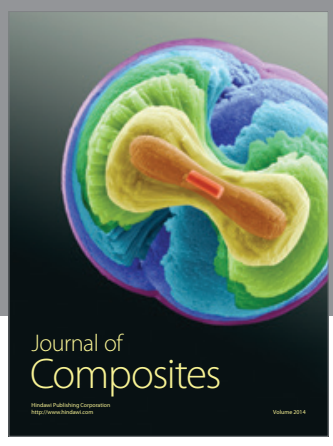
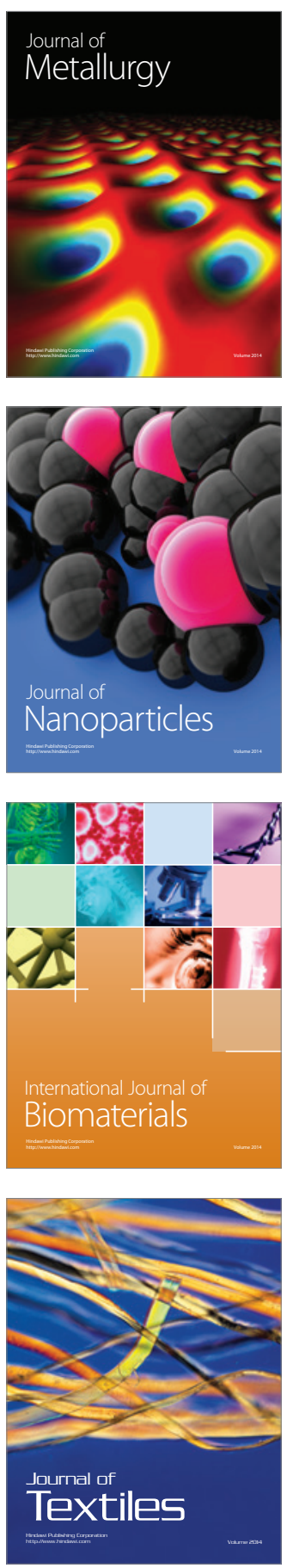